\title{
Publisher Correction: Pathologist-level interpretable whole-slide cancer diagnosis with deep learning
}

Zizhao Zhang, Pingjun Chen D, Mason McGough, Fuyong Xing, Chunbao Wang, Marilyn Bui, Yuanpu Xie, Manish Sapkota, Lei Cui, Jasreman Dhillon, Nazeel Ahmad, Farah K. Khalil, Shohreh I. Dickinson, Xiaoshuang Shi, Fujun Liu, Hai Su, Jinzheng Cai and Lin Yang

Correction to: Nature Machine Intelligence https://doi.org/10.1038/s42256-019-0052-1, published online 13 May 2019.

In the version of this Article originally published, both the sixth and seventh $x$-axis labels in Fig. 6a incorrectly said 0.5 . They should have said 0.6 and 0.7 , respectively. This has now been corrected.

Published online: 17 July 2019

https://doi.org/10.1038/s42256-019-0082-8 